\title{
On the satellite altimeter crossover problem
}

\author{
H. van Gysen ${ }^{1}$, R. Coleman ${ }^{2,3}$ \\ ${ }^{1}$ Department of Surveying and Mapping, University of Natal, King George V Avenue, Durban. 4001. South Africa \\ ${ }^{2}$ Department of Surveying and Spatial Information Science, University of Tasmania, GPO Box 252-76, Hobart. 7001. TAS. Australia \\ ${ }^{3}$ CSIRO Division of Oceanography, GPO Box 1538, Hobart. 7001. TAS. Australia
}

Received 11 September 1995; Accepted 2 September 1996

\begin{abstract}
Mean sea surface heights and residual radial orbit errors are estimated simultaneously in a single global crossover adjustment of multiple cycles of satellite altimetry data. The rank defect inherent in the estimation problem is explicitly identified and treated in various ways to give solutions that minimise (in norm) either orbit errors or mean sea surface heights. The rank defect gives rise to geographically correlated orbit error, consisting of those components of the orbit error or those components of the map of sea surface heights which fall within the nullspace of the estimation problem and which cannot be distinguished as orbit error or ocean signal. We show that, in the case of Topex/ PoSEIDON data, the geographically correlated error consists largely of long-wavelength and long-period sea surface fluctuations, which in the past has often been assigned as orbit error.
\end{abstract}

\section{Introduction}

The Topex/Poseidon (T/P) satellite altimeter mission saw a quantum improvement in the accuracy of altimeter sea surface heights (SSHs) due, in large measure, to improved orbits. T/P has an orbit error budgeted to be $13 \mathrm{~cm}$ (Topex/Poseidon Project, 1992), and reported in reality to be better than $5 \mathrm{~cm}$ (Le Traon et al., 1994; Tapley et al., 1994), in contrast to Geosat orbits, which were initially good to 3 metres and improved to about $20-50 \mathrm{~cm}$ for the precise orbits based on the GEM-T2 geopotential model (Shum et al., 1990; Haines et al., 1994).

With such good orbits and consequently such good measured SSHs, it is argued in some quarters it does more harm than good to use any of the empirical, nondynamical orbit error reduction techniques that have become a traditional part of satellite altimetry data

Correspondence to: $\mathrm{H}$. van Gysen processing (Stammer and Wunsch, 1994). Of particular concern are the indications that orbit error removal also removes long-wavelength, basin-scale and larger, ocean signals (Tai, 1991; Miller et al., 1993; Tai and Wagner, 1994; Wagner and Tai, 1995). We show here that this concern is well founded, and that supposed orbit errors, certainly in the case of $\mathrm{T} / \mathrm{P}$ orbits, in reality contain long-wavelength, small-amplitude ocean signals.

On the other hand, the reduction of noise levels in SSHs allows other signals to be unmasked, such as planetary waves, shortcomings in present tide models, and annual and longer period changes in mean sea level (MSL). It is our view that if these long-wavelength, small-amplitude effects are to be uncovered, care is still needed in the reduction and processing of altimetry data. The main requirement is to estimate mean sea level in a consistent and uniform way since everything else flows from this mean - mesoscale variability, seasonal variations in sea surface height (SSH), trend in mean sea level (Wagner and Cheney, 1992) - while taking into account the radial orbit error, including the geographically correlated component of the error. Further, in the processing of ERS-1/2 data and in the reprocessing of Geosat data, improved gravity field models notwithstanding, there is still a need to take residual radial orbit errors into account.

In this paper we present a model for doing a global crossover adjustment in which the mean sea surface heights are estimated directly, and not removed at the outset through crossover differences, as is usually done. Also part of the model is an estimation of the radial component of the orbit error, for which any of the models which have gained acceptance in the last few years can be used. We will concentrate on an orbit error model (in equation (1) below) that derives from the analytical studies by Colombo (1984), Wagner (1985), Rosborough (1986), Engelis (1988), Moore and Rothwell (1990), and others, which takes into account errors in the geopotential, errors in the modelling of drag and solar radiation forces on the satellite, and errors in the initial state vector. 
Whatever model is chosen to represent the orbit error, it will have associated with it a null surface which, on the basis of the given altimeter measurements alone, cannot be included in the mean sea surface or assigned to the orbit error. This is the geographically correlated error (GCE) falling within the nullspace of the estimation problem; the dimension of the surface equals the dimension of the nullspace (Schrama, 1989; 1992). The treatment of this rank defect requires special attention. It can be determined (in whole, or perhaps only in part) by outside measurements or by outside constraints, for example, in the comparison of the T/P orbits (based on the JGM-2 gravity field) with orbits obtained using the T/P GPS Demonstration receiver (Bertiger et al., 1994), and in the $T / P$ verification measurements at Platform Harvest, Lampione and Bass Strait (Christensen et al., 1994; Menard et al., 1994; White et al., 1994). In the absence of any external reference, the singularity of the estimation problem needs to be overcome in some other way. This has often been done in an ad hoc fashion, by using master arcs, or by putting extra weight on the diagonal (ridging) of the normal equation matrix of the crossover difference problem (Rapp, 1979; Douglas et al., 1984; Tai, 1988; Houry et al., 1994). Alternate approaches have been suggested by, for example, Wunsch and Zlotnicki (1984) and Blanc et al. (1995) using inverse formalism. However, this latter technique, while making a much better attempt to separate ocean signal from radial orbit error and altimeter measurement noise, remains sub-optimal as global analyses cannot be attempted due to the computational burden of the problem.

In our approach we have addressed the following issues: (i) to make global solutions computationally tractable, (ii) using a well-established analytical form for the description of the radial orbit error model, the exact nature of the rank defect of the problem can be formulated, (iii) a conscious choice can then be made as to where to 'place' the nullspace of the solution.

The choice of nullspace definition can be guided by background knowledge of the ocean signal and the likely magnitudes of mean orbit errors, but in this definition there can be no absolute certainty about where the GCE belongs. If one believes the GCE indeed to be orbit error, a solution can be found for the mean SSHs which contains nothing that looks like orbit error. At the other extreme, if one believes that the orbits are free of GCE, a solution can be found in which the overall change to the orbits is zero and the GCE signature is placed in the SSHs. It is also possible to obtain solutions which distribute the nullspace between orbits and SSHs in some predetermined proportion, or which assign certain components of the nullspace to $\mathrm{SSH}$ and the remainder as a correction to the orbits overall.

If the choice as to what to do with the GCE is ultimately arbitrary, it should also be mentioned that all the possibilities suggested above will be 'minimum constraint' solutions for which the measurement residuals will be invariant. Thus, for studies in which the residuals are used - for SSH variability, seasonal variability, long-term trends - it does not matter which solution is chosen. It does matter, however, if the SSHs themselves are used, to determine general ocean circulation, for example.

The advantages we see for our approach are the following:

- By working directly with SSHs, and not with crossover differences, there is no need to assume an invariant sea level change over the interval making up the crossover pair, i.e., no time criterion for a minimum crossover time between pairs of tracks need be applied.

- The mean sea surface is an explicit average over the number of repeat data in the adjustment, with all cycles contributing simultaneously; moreover, the estimation of mean SSH is simultaneous with estimation of the orbit error parameters.

- The nullspace of the estimation problem is explicitly characterised, and is accounted for (taken up in the orbits or in the mean SSHs) in a deliberate way.

- And finally, the solution presented here is computationally tractable, making possible crossover solutions for full or multiple altimetric missions.

\section{Theory}

\subsection{Orbit Model}

Suppose a satellite to be in a repeating, near-circular orbit, with orbital period $T_{0}$ and $T_{e}$ the length of a nodal day (the rotation period of the earth with respect to the satellite's orbital plane); then $N_{R} T_{0}=N_{D} T_{e}$ for integers $N_{R}$ and $N_{D}$ relatively prime to each other. (The satellite thus completes $N_{R}$ orbits in $N_{D}$ whole nodal days; for T/ $\mathrm{P}, N_{R}=127, N_{D}=10$ ).

As indicated in Figure 1, $i$ is the inclination of the satellite's orbit, $\omega$ the argument of perigee, $\Omega$ the right ascension of the ascending node, $\theta$ sidereal time, and $M$ is the mean anomaly. For satellites that are in a 'frozen' repeat orbit, or in an orbit close to critical inclination, $\dot{\omega}=0$ and $\bar{\omega}=\pi / 2$. T/P has a frozen orbit close to critical inclination. Further, we shall use the notation $\omega_{0}=\omega+M, \omega_{e}=\Omega-\theta$ (so that $\dot{\omega}_{e} / \dot{\omega}_{0}=-N_{D} / N_{R}$ ).

The model for the radial orbit error we shall consider has two components (after Moore and Ehlers (1993)):

$\Delta r=\Delta r_{n g}+\Delta r_{g}$,

where

$$
\begin{aligned}
\Delta r_{n g}= & D_{1}\left(t-t_{0}\right)+D_{2}\left(t-t_{0}\right) \sin M \\
& +D_{3}\left(t-t_{0}\right) \cos M+D_{4}\left(t-t_{0}\right) \sin 2 M \\
& +D_{5}\left(t-t_{0}\right) \cos 2 M \\
\Delta r_{g}= & \sum_{l=0}^{l_{\max }} \sum_{m=0}^{l} \sum_{p=0}^{l}\left(A_{l m p} \cos \psi_{l m p}+B_{l m p} \sin \psi_{l m p}\right)
\end{aligned}
$$

where $t$ is time, $t_{0}$ is a reference epoch, $\psi_{l m p}=(l-2 p) \omega_{0}$ $+m \omega_{e}=\dot{\psi}_{l m p} t=\dot{\omega}_{0}\left(l-2 p-m N_{D} / N_{R}\right) t, \quad$ and $A_{l m p}$, 
$B_{l m p}, D_{k}$ are coefficients. The $\Delta r_{n g}$ term takes into account error contributions that are mostly nongravitational, such as initial state vector and surface force modelling (Moore and Rothwell, 1990; Moore and Ehlers, 1993), and $\Delta r_{g}$ contains errors due to the gravity field. Notice that the bias term is the first term in the summation for $\Delta r_{g}$.

The 'gravitational' frequencies $\dot{\psi}_{\text {lmp }}$ in equation (3) account for errors in the gravitational field; the primary term is at one cycle/rev, the other terms are harmonics of this fundamental frequency, $\dot{\omega}_{0}$, and combinations of $\dot{\omega}_{0}$ with the nodal frequency $\dot{\omega}_{e}$. These are the dominant frequencies predicted by analytical perturbation theory and are shown up by spectral analysis of orbit errors (see, for example Fu and Vazquez (1988); Chelton and Schlax (1993), Houry et al. (1994), among many such studies).

The terms in equation (3) are related to surface spherical harmonics by Kaula's transformation (Kaula, 1966):

$$
\begin{aligned}
{\left[\begin{array}{l}
r_{l m}^{c} \\
r_{l m}^{s}
\end{array}\right]=} & {\left[\begin{array}{l}
C_{l m} P_{l m}(\sin \phi) \cos m \lambda \\
S_{l m} P_{l m}(\sin \phi) \sin m \lambda
\end{array}\right] } \\
= & {\left[\begin{array}{c}
C_{l m} \sum_{p=0}^{l} F_{l m p}(i)\left[\begin{array}{c}
\cos \psi_{l m p} \\
\sin \psi_{l m p}
\end{array}\right]_{l-m \text { odd }}^{l-m \text { even }} \\
S_{l m} \sum_{p=0}^{l} F_{l m p}(i)\left[\begin{array}{c}
\sin \psi_{l m p} \\
-\cos \psi_{l m p}
\end{array}\right]_{l-m \text { odd }}^{l-m \text { even }}
\end{array}\right] }
\end{aligned}
$$

where $\phi$ is geocentric latitude, $\lambda$ is longitude, $P_{l m}$ are associated Legendre functions and the $F_{l m p}(i)$ are the inclination functions given by Kaula (1966).

When we sum

$\Delta r_{g}=\sum_{l=0}^{l_{\max }} \sum_{m=0}^{l}\left(r_{l m}^{c}+r_{l m}^{s}\right)$ with

$$
\left[\begin{array}{c}
A_{l m p} \\
B_{l m p}
\end{array}\right]=F_{l m p}(i)\left[\begin{array}{c}
{\left[\begin{array}{c}
C_{l m} \\
-S_{l m}
\end{array}\right]_{l-m \text { odd }}^{l-m \text { even }}} \\
{\left[\begin{array}{c}
S_{l m} \\
C_{l m}^{l-m \text { even }}
\end{array}\right]_{l-m \text { odd }}}
\end{array}\right],
$$

we see that the expression for the 'gravitational' component of the radial error is transformed from the time domain to the spatial or geographical domain. Each spherical harmonic $r_{l m}^{c}, r_{l m}^{s}$ with $l \leq l_{\max }$ can be expressed as a linear combination $l$ orbital frequencies (plus a possible 'zero' frequency). For $l_{\max }=2$, these linear combinations are (see also Schrama (1989)):

$$
\begin{aligned}
r_{00}^{c}= & C_{00} F_{000} \\
r_{10}^{c}= & C_{10}\left(F_{100}-F_{101}\right) \sin \omega_{0} \\
r_{11}^{c}= & C_{11}\left[F_{110} \cos \left(\omega_{0}+\omega_{e}\right)+F_{111} \cos \left(\omega_{0}-\omega_{e}\right)\right] \\
r_{11}^{s}= & S_{11}\left[F_{110} \sin \left(\omega_{0}+\omega_{e}\right)-F_{111} \sin \left(\omega_{0}-\omega_{e}\right)\right] \\
r_{20}^{c}= & C_{20}\left[F_{201}+\left(F_{200}+F_{202}\right) \cos 2 \omega_{0}\right] \\
r_{21}^{c}= & C_{21}\left[F_{210} \sin \left(2 \omega_{0}+\omega_{e}\right)+F_{211} \sin \omega_{e}\right. \\
& \left.-F_{212} \sin \left(2 \omega_{0}-\omega_{e}\right)\right] \\
r_{21}^{s}= & S_{21}\left[-F_{210} \cos \left(2 \omega_{0}+\omega_{e}\right)-F_{211} \cos \omega_{e}\right. \\
& \left.-F_{212} \cos \left(2 \omega_{0}-\omega_{e}\right)\right] \\
r_{22}^{c}= & C_{22}\left[F_{220} \cos \left(2 \omega_{0}+2 \omega_{e}\right)+F_{221} \cos 2 \omega_{e}\right. \\
& \left.+F_{222} \cos \left(2 \omega_{0}-2 \omega_{e}\right)\right] \\
r_{22}^{s}= & S_{22}\left[F_{220} \sin \left(2 \omega_{0}+2 \omega_{e}\right)+F_{221} \sin 2 \omega_{e}\right. \\
& \left.-F_{222} \sin \left(2 \omega_{0}-2 \omega_{e}\right)\right] .
\end{aligned}
$$

These linear combinations, the 'geographic correlations', are what give rise to the GCE and the singularity in the crossover adjustment problem. The nature of these singularities is discussed further in section 2.3 below.

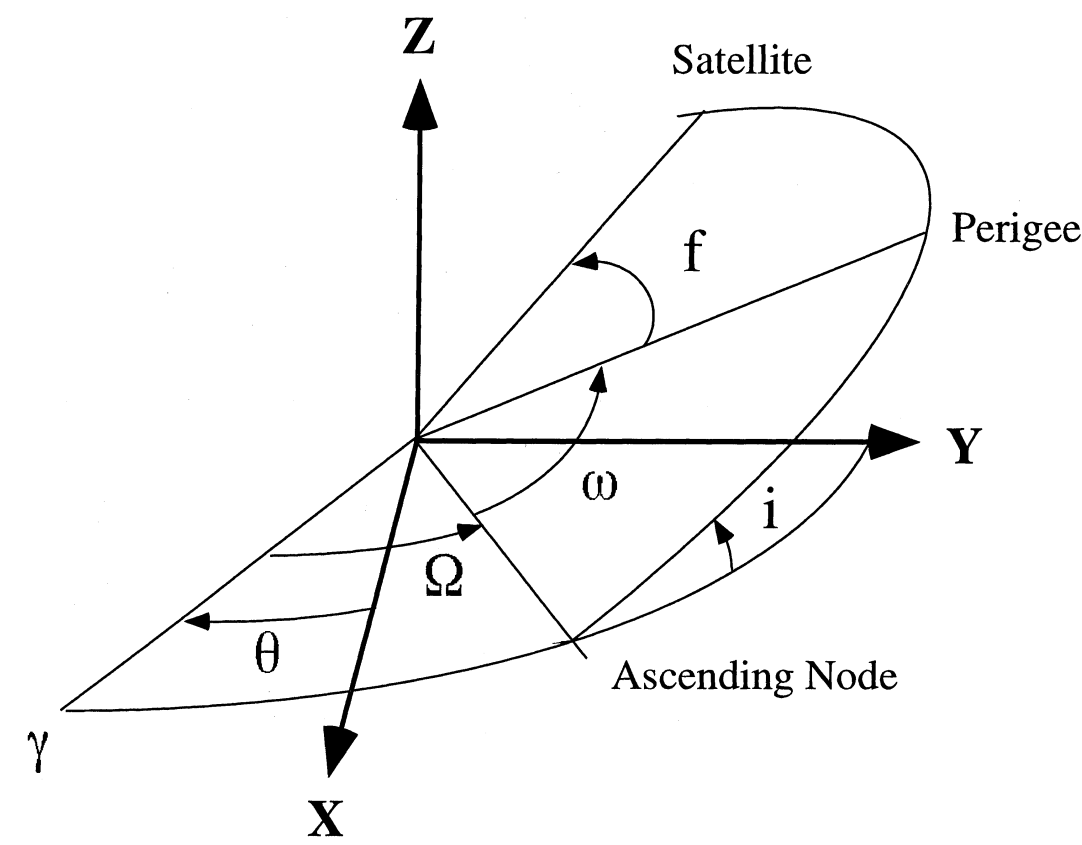

Fig. 1. Relation between orbital elements: $i$ is inclination of the orbit, $\omega$ argument of perigee, $\Omega$ right ascension of the ascending node, $\theta$ sidereal time, $M$ mean anomaly 


\subsection{Altimetric Equation}

Let crossover points (crossovers of ascending and descending passes within a single $N_{D}$-day repeat cycle) be numbered or ordered in some way; we will use the absence or presence of primes to distinguish between ascending and descending passes. At the $i$ th crossover point the altimetric equation relating altimeter measurement and sea surface height is (for the ascending pass):

$l_{i}=h_{i}+A_{i} x+\epsilon_{i}$

where $l_{i}=r_{\text {ellipsoid }}-r_{\text {altimeter }}$ is the difference between the height (range) of the satellite above the reference ellipsoid and the measured range to the instantaneous sea surface, with the altimeter range corrected in the usual way for such effects as earth and ocean tides, atmospheric pressure, significant wave height and propagation delays; $h_{i}$ is the instantaneous $\mathrm{SSH}$ above the ellipsoid at the crossover point; $A_{i} x$ the orbital correction term, and $\epsilon_{i}$ is measurement noise. The instantaneous SSH can be split as

$h_{i}=\bar{h}_{i}+\zeta_{\text {instant }, \mathrm{i}}=N_{i}+\zeta_{\text {permanent }, i}+\zeta_{\text {instant }, i}$,

where $\bar{h}_{i}$ is the mean $\mathrm{SSH}\left(=N_{i}\right.$, the geoid height plus $\zeta_{\text {permanent }, i}$, the permanent or stationary component of the sea surface topography (SST)), and $\zeta_{\text {instant, } i}$ is the instantaneous or variable component of SST.

The $\bar{h}_{i}$ term is used since it is not possible to separate $N_{i}$ and $\zeta_{\text {permanent, } i}$ unless we have additional information or use some form of external constraints. Some possibilities are: (1) use inverse formalisms such as those originally proposed by Wunsch and Gaposchkin (1980); (2) use simultaneous, in-situ hydrographic measurements in the set of crossover observation equations (van Gysen et al. (1995)) or forms of oceanographic constraint equations in closed areas, such as conservation of horizontal divergence - for example, Mesais and Strub (1995)); (3) adopt a model for $\zeta_{\text {permanent }, i}$ and thus solve for $N_{i}$ - this gives us no new oceanographic information, only a geoid that is as good as the definition of $\zeta_{\text {permanent, } i}$; (4) wait for the launch of a dedicated geopotential research satellite mission. To obtain new oceanographic results, the last two options are not viable possibilities and so we adopt a pragmatic view towards a solution and use our proposed crossover technique.

We will combine $\zeta_{\text {instant, } i}$ and $\epsilon_{i}$ into a single 'error' term, $v_{i}$, so that our observation model becomes

$l_{i}=\bar{h}_{i}+A_{i} x+v_{i}$.

The $v_{i}$ term thus contains both measurement error and the time-varying, instantaneous component of SST. The measurement equation for the descending pass at the same point reads

$l_{i}^{\prime}=\bar{h}_{i}+A_{i}^{\prime} x+v_{i}^{\prime}$.

At this point it is customary to form crossover differences, $l_{i}-l_{i}^{\prime}$, in which the mean $\mathrm{SSH}, \bar{h}_{i}$, no longer appears. However, our approach is to continue with the measurement model above, in which both mean SSH and orbit correction terms appear.

Suppose there are a total of $n$ crossovers; putting $n$ ascending measurements $l_{i}$ and $n$ descending measurements $l_{i}^{\prime}$ together, we have

$$
\begin{aligned}
l & =\bar{h}+A x+v \\
l^{\prime} & =\bar{h}+A^{\prime} x+v^{\prime}
\end{aligned}
$$

where $l, l^{\prime}, v, v^{\prime}$ and $\bar{h}$ are $n$-vectors, $x$ is a $u$-vector of (unknown) orbit model coefficients, and $A, A^{\prime}$ are $(n, u)$ matrices of observation equation coefficients. We assume all $2 n$ measurements are of equal precision and uncorrelated. This is a considerable presumption, since the errors $v$ and $v^{\prime}$ also contain $\mathrm{SSH}$ variations, which are markedly inhomogeneous and can be significantly correlated, both spatially and temporally.

From these $2 n$ measurements we seek to estimate the $n$ heights in $\bar{h}$ and $u$ orbit parameters in $x$; for this we shall use least squares. However, a problem to be faced is that the estimation problem in equation (12) is singular, because of the GCE. It is to the identification and regularisation of this singularity that we now turn.

\subsection{Singularity}

Another way of writing equation (12) is as

$$
\left[\begin{array}{l}
l \\
l^{\prime}
\end{array}\right]=X\left[\begin{array}{l}
\bar{h} \\
x
\end{array}\right]+\left[\begin{array}{c}
v \\
v^{\prime}
\end{array}\right]
$$

where

$X_{(2 n, n+u)}=\left[\begin{array}{ll}I_{n} & A \\ I_{n} & A^{\prime}\end{array}\right]$

and $I_{n}$ is the $(n, n)$ identity matrix.

Let $t_{i}$ be the time of the ascending pass measurement at the $i$ th crossover point, $t_{i}^{\prime}$ the time of the descending pass measurement at the same point, and $\left(\phi_{i}, \lambda_{i}\right)$ its (geocentric) latitude and longitude. (Note that the latitudes on the Geodetic Data Records are geodetic latitudes for the T/P reference ellipsoid (Benada, 1993).) Let $\psi_{l m p, i}=\dot{\psi}_{l m p} t_{i}$ be orbital angles corresponding to the times of ascending pass measurements, and $\psi_{\text {lmp }, i}^{\prime}=\dot{\psi}_{\text {lmp }} t_{i}^{\prime}$ be orbital angles corresponding to descending crossover times.

In equation (4) it was seen that the spherical harmonics $r_{l m}^{c}, r_{l m}^{s}$ evaluated at $\left(\phi_{i}, \lambda_{i}\right)$ can be expressed as linear combinations of $l+1$ elements in turn from the set $\left\{\cos \psi_{l m p, i}, \sin \psi_{l m p, i}: p=0,1, \ldots, l\right\}$ at ascending crossover times. The identical linear combinations also relate $r_{l m}^{c}, r_{l m}^{s}$ to the set $\left\{\cos \psi_{l m p, i}^{\prime}, \sin \psi_{l m p, i}^{\prime}: p=\right.$ $0,1, \ldots, l\}$ at descending crossover times, and further, the same linear combinations hold for all $i=1, \ldots, n$. Each pair $\left(r_{l m}^{c}, r_{l m}^{s}\right)$ (one only if $m=0$ ) thus gives rise to two linear dependencies in the columns of $X$, between its first $n$ columns and the $u$ columns of $\left[\begin{array}{l}A \\ A^{\prime}\end{array}\right]$.

There are $r=\left(l_{\max }+1\right)^{2}$ spherical harmonics of degree less than or equal to $l_{\max }$, and therefore $r$ such 
linear dependencies among the columns of $X$. Moreover, since the $r$ spherical harmonics are independent basis functions (none is a linear combination of the remaining $r-1$ harmonics), the $r$ combinations expressing the linear dependencies are themselves linearly independent. That is to say, there is a matrix $G_{(n+u, r)}$ such that $X G=0_{(2 n, r)}$ and $\operatorname{rank}(G)=r$. The matrix $G$ is not unique. If, in our example with $l_{\max }=2$, the 26 orbit parameters were to be ordered as $\left(D_{1}, D_{2}, D_{3}, D_{4}\right.$, $D_{5}, A_{000}, A_{100}, A_{110}, A_{111}, A_{200}, A_{210}, A_{211}, A_{212}, A_{220}$, $\left.A_{221}, A_{222}, B_{100}, B_{110}, \ldots, B_{222}\right)$, one way in which $G$ could be written would be as $(u=26, r=9)$

$G=\left[\begin{array}{c}G_{1_{(n, 9)}} \\ G_{2_{(26,9)}}\end{array}\right]$,

where $G_{1}$ has as its $i$ th row the 9 -vector $\left(1, \sin \phi_{i}\right.$, $\cos \phi_{i} \cos \lambda_{i}, \cos \phi_{i} \sin \lambda_{i}, \frac{3}{2} \sin ^{2} \phi_{i}-\frac{1}{2}, 3 \sin \phi_{i} \cos \phi_{i} \cos \lambda_{i}$, $\left.3 \sin \phi_{i} \cos \phi_{i} \sin \lambda_{i}, 3 \cos ^{2} \phi_{i} \sin 2 \lambda_{i}\right)$, of spherical harmonic basis functions evaluated at $\left(\phi_{i}, \lambda_{i}\right)$. The matrix $G_{2}$ has the form

$G_{2}=\left[\begin{array}{c}0_{(5,9)} \\ -G_{21_{(21,9)}}\end{array}\right]$,

where $G_{21}$ has coefficients $F_{l m p}(i)$ as on the right hand side of equations (7). In fact, another way of writing equations (7) would be as

$$
\begin{aligned}
G_{1}+A G_{2} & =0 \\
G_{1}+A^{\prime} G_{2} & =0
\end{aligned}
$$

equivalent to $X G=0$. The block of zeroes appears in $G_{2}$ because the terms in $\left(t-t_{0}\right),\left(t-t_{0}\right) \sin M,\left(t-t_{0}\right)$ $\cos M,\left(t-t_{0}\right) \sin 2 M$ and $\left(t-t_{0}\right) \cos 2 M$ in equation (2) do not contribute to the rank defect.

The nullspace of $X$ thus has rank at least $r=\left(l_{\max }+1\right)^{2}$. At the same time it has rank at most $r$ : should the $r$ basis functions not span the nullspace, the nullspace will contain spherical harmonics of degree greater than $l_{\max }$, which will imply (in an extension of equation (7)) that the orbit error model contains frequencies not chosen to be part of the model. Since those frequencies are not part of the model, the corresponding spherical harmonics cannot be part of the GCE either. (An essential point to note is that the singularity in the crossover adjustment problem depends on the functional form chosen for the radial orbit error model, and not on what that error 'really' is.)

\subsection{Datum Constraints}

\subsubsection{Minimum norm}

The normal equations corresponding to the least squares estimation problem in equations (12) or (13) are

$$
\left[\begin{array}{cc}
2 I_{n} & A+A^{\prime} \\
\left(A+A^{\prime}\right)^{T} & A^{T} A+A^{\prime T} A^{\prime}
\end{array}\right]\left[\begin{array}{l}
\bar{h} \\
x
\end{array}\right]=\left[\begin{array}{c}
l+l^{\prime} \\
A^{T} l+A^{\prime T} l^{\prime}
\end{array}\right]
$$

where the normal equation matrix is singular, with a rank defect which we have seen is equal to $r=\left(l_{\max }+1\right)^{2}$, so that there are a manifold of solutions satisfying the least squares condition (minimising $\left.v^{T} v+v^{T} v^{\prime}\right)$. One particular solution is the minimum norm solution (minimising $\bar{h}^{T} \bar{h}+x^{T} x$ ), given by the pseudoinverse of $X$, or by the solution of the (nonsingular) equation system

$$
\begin{aligned}
& {\left[\begin{array}{ccc}
2 I_{n} & A+A^{\prime} & G_{1} \\
\left(A+A^{\prime}\right)^{T} & A^{T} A+A^{\prime T} A^{\prime} & G_{2} \\
G_{1}^{T} & G_{2}^{T} & 0_{(r, r)}
\end{array}\right]\left[\begin{array}{c}
\bar{h} \\
x \\
k
\end{array}\right]} \\
& =\left[\begin{array}{c}
l+l^{\prime} \\
A^{T} l+A^{\prime T} l^{\prime} \\
0_{(r, 1)}
\end{array}\right],
\end{aligned}
$$

where $k$ is an $r$-vector of Lagrange multipliers. The solution distributes the GCE between mean SSHs and orbits in a way that minimises the parameter norm. The constraints that have in this case been added to the normal equations (14) are

$G_{1}^{T} \bar{h}+G_{2}^{T} x=0$.

\subsubsection{SSH constraints}

If one believes that the GCE is due to orbit error and belongs to the orbits and not to SSHs, then the mean heights $\bar{h}$ can be made to be free of anything that looks like GCE, free of anything that belongs to the nullspace of the estimation problem, by adding to the normal equations (14) the constraints

$G_{1}^{T} \bar{h}=0$.

With these constraints the mean SSH at the crossover points will contain no spherical harmonic that belongs to the GCE and any GCE will be placed in the orbits. For example, for $l \leq l_{\max }$,

$\sum_{i=1}^{n} P_{l m}\left(\sin \phi_{i}\right) \cos m \lambda_{i} \cdot \bar{h}_{i}=0$,

since the $P_{l m}\left(\sin \phi_{i}\right) \cos m \lambda_{i}$ make up a row of $G_{1}^{T}$.

It must be made clear that if this constraint is used, any component of the altimeter data of spherical harmonic degree less than or equal to $l_{\max }$ that properly belongs to $\bar{h}$ must first be removed. Certainly this means that the relevant geoid and SST fields need to be removed. Though the spherical harmonic representation of the geoid contains no terms of degree 0 or 1 , and also $C_{20}=0$ (Heiskanen and Moritz, 1967, p. 217), the terms that remain, $C_{21}, S_{21}, C_{22}, S_{22}$, are quite significant, giving the largest-scale features of the geoid. Similarly, the sea surface topography also contains significant terms of degree $l \leq l_{\max }$ (Tai, 1983), and these also need to be removed. Any remaining error in the geoid or SST of degree less than or equal to $l_{\max }$ is indistinguishable from orbit error, and will be taken to be part of the GCE, thereby being placed in the orbits. 
When constraints of this kind are considered, the time-varying component of the GCE can be determined (albeit not the mean component). It will be worth looking at this variation, since we will show below that the signal largely coincides with the variable component of the SST at the spatial scales of the GCE, the gravitational errors at these scales being constant over the mission period. Another way of considering this is that at certain periods the recovered GCE is unlikely to be orbit error, for example, at semi-annual or annual time scales. The global crossover adjustment process extracts from the altimeter measurement those frequencies that are contained in the specified orbital frequencies of equation (3), except those that fall within the nullspace of the problem.

\subsubsection{Orbit constraints}

On the other hand, if one believes that the GCE belongs to the ocean surface and not to the orbits, the orbits can be made to be free of anything that looks like GCE at the frequencies of equation (3), by enforcing the constraints

$G_{2}^{T} x=0$

on the normal equations (14). With these constraints the GCE is placed entirely in the SSHs.

It is also possible to weight the play-off between the two sets of constraints, or to assign some constraints to SSHs and the remainder to the orbits.

We will implement the orbit constraints in equation (18), since this is the easier solution to calculate, and comes closes in spirit to the usual crossover difference solution. Other minimum constraint solutions can then be found using similarity transformations.

\subsection{Solution for Single Repeat Cycle}

\subsubsection{Orbit constraints}

The normal matrix $X^{T} X$ in equation (14) can be reduced by crossover differencing to

$$
\left[\begin{array}{cc}
2 I_{n} & A+A^{\prime} \\
0 & \frac{1}{2}\left(A-A^{\prime}\right)^{T}\left(A-A^{\prime}\right)
\end{array}\right] .
$$

The rank defect is now located in the sub-matrix $\frac{1}{2}\left(A-A^{\prime}\right)^{T}\left(A-A^{\prime}\right)$, which has rank $n-r$. Moreover, $\frac{1}{2}\left(A-A^{\prime}\right)^{T}\left(A-A^{\prime}\right) G_{2}=0$; hence the pseudoinverse of $\frac{1}{2}\left(A-A^{\prime}\right)^{T}\left(A-A^{\prime}\right)$ is (cf. Leick (1990), p. 102).

$$
\begin{aligned}
Q_{x}= & \left\{\frac{1}{2}\left(A-A^{\prime}\right)^{T}\left(A-A^{\prime}\right)+G_{2} G_{2}^{T}\right\}^{-1} \\
& -G_{2}\left(G_{2}^{T} G_{2}\right)^{-2} G_{2}^{T}
\end{aligned}
$$

(So that $G_{2}^{T} Q_{x}=0$.) Hence

$$
x=\frac{1}{2} Q_{x}\left(A-A^{\prime}\right)^{T}\left(l-l^{\prime}\right)
$$

$\bar{h}=\frac{1}{2}\left(l+l^{\prime}-\left(A+A^{\prime}\right) x\right)$.

or

$$
\left[\begin{array}{l}
\bar{h} \\
x
\end{array}\right]_{\text {orbit constrained }}=X^{g}\left[\begin{array}{l}
l \\
l^{\prime}
\end{array}\right],
$$

where $X^{g}$ is the generalised inverse of $X$ associated with the normal equations reduced in the way above:

$X^{g}=\frac{1}{2}\left[\begin{array}{c}{\left[\begin{array}{c}I_{n} \\ I_{n}\end{array}\right]^{T}-\frac{1}{2}\left(A+A^{\prime}\right) Q_{x}\left[\begin{array}{c}A-A^{\prime} \\ -\left(A-A^{\prime}\right)\end{array}\right]^{T}} \\ Q_{x}\left[\begin{array}{c}A-A^{\prime} \\ -\left(A-A^{\prime}\right)\end{array}\right]^{T}\end{array}\right]$

( $X^{g}$ is a 'right weak generalised inverse' of $X$ (Boullion and Odell, 1971).) The cofactor matrix of this solution for $\bar{h}$ and $x$ is

$$
\begin{aligned}
X^{g} X^{g^{T}} & =\left[\begin{array}{cc}
Q_{h} & Q_{h x} \\
Q_{x h} & Q_{x}
\end{array}\right]_{\text {orbit constrained }} \\
& =\frac{1}{2}\left[\begin{array}{cc}
I_{n}+\frac{1}{2}\left(A+A^{\prime}\right) Q_{x}\left(A+A^{\prime}\right)^{T} & -\left(A+A^{\prime}\right) Q_{x} \\
-Q_{x}\left(A+A^{\prime}\right)^{T} & 2 Q_{x}
\end{array}\right] .
\end{aligned}
$$

We re-iterate: the orbit constraint $G_{2}^{T} x=0$ applied in obtaining the solution of equation (22) presumes that the GCE properly belongs to $\bar{h}$.

\subsubsection{SSH constraints}

The solution for SSHs and orbit parameters in which the SSHs are constrained by $G_{1}^{T} \bar{h}=0$ may be obtained from the solution of the normal equations with the normal matrix $X^{T} X$ reduced to, with $N_{22}=\left(A^{T} A+A^{\prime T} A^{\prime}\right)$,

$$
\left[\begin{array}{cc}
2 I_{n}-\left(A+A^{\prime}\right) N_{22}^{-1}\left(A+A^{\prime}\right)^{T} & 0 \\
\left(A+A^{\prime}\right)^{T} & N_{22}
\end{array}\right],
$$

starting with the pseudoinverse of the top-left submatrix, with rank $n-r$, and for which

$$
\left\{2 I_{n}-\left(A+A^{\prime}\right) N_{22}^{-1}\left(A+A^{\prime}\right)^{T}\right\} G_{1}=0
$$

(so that $G_{1}^{T} Q_{h}=0$ as required, where $Q_{h}$ is the pseudoinverse of $\left.2 I_{n}-\left(A+A^{\prime}\right) N_{22}^{-1}\left(A+A^{\prime}\right)^{T}\right)$.

Rather than calculate the solution in this way (since the number of crossover points is likely to exceed by far the number of crossover parameters, $n \gg u$ ), we prefer to calculate the solution by transforming the results in equations (20) and (21):

$$
\left[\begin{array}{l}
\bar{h} \\
x
\end{array}\right]_{\mathrm{SSH} \text { constrained }}=T\left[\begin{array}{l}
\bar{h} \\
x
\end{array}\right]_{\text {orbit constrained }},
$$

and

$X_{\mathrm{SSH} \text { constrained }}^{g}=T X_{\text {orbit constrained }}^{g}$, 
where (cf. Leick (1990), p. 103):

$T=I_{n+u}-\left[\begin{array}{ll}G_{1}\left(G_{1}^{T} G_{1}\right)^{-1} G_{1}^{T} & 0 \\ G_{2}\left(G_{1}^{T} G_{1}\right)^{-1} G_{1}^{T} & 0\end{array}\right]$.

The cofactor matrix for the transformed solution is

$$
\begin{gathered}
X^{g} X_{\mathrm{SSH} \text { constrained }}^{g^{T}}=\left[\begin{array}{cc}
Q_{h} & Q_{h x} \\
Q_{x h} & Q_{x}
\end{array}\right]_{\mathrm{SSH} \text { constrained }} \\
=T\left(X^{g} X_{\text {orbit constrained }}^{g^{T}}\right) T^{T} .
\end{gathered}
$$

What equation (27) does, in effect, is to remove from the mean SSHs $\bar{h}$ the component that looks like GCE, and add that component to the orbit error.

The columns of $G_{1}$ are spherical harmonic basis functions evaluated at crossover points. These points are in the ocean, and there are rather more of them in the Southern Hemisphere than in the Northern Hemisphere. The basis functions thus evaluated will therefore not be orthogonal, i.e., $G_{1}^{T} G_{1}$ as used in equation (27) will not be a diagonal matrix, and there will be leakage or aliasing between the different functions in $G_{1}$, and consequently also between the orbital frequency terms in the orbit model of equation (3).

\subsubsection{Minimum norm solution}

The minimum norm solution can also be obtained from the solution in equation (22) in which the orbit has been constrained:

$X^{+}=X_{\text {minimum norm }}^{g}=T X_{\text {orbit constrained }}^{g}$,

where, with $\bar{G}=G_{1}^{T} G_{1}+G_{2}^{T} G_{2}$,

$T=I_{n+u}-\left[\begin{array}{ll}G_{1} \bar{G}^{-1} G_{1}^{T} & G_{1} \bar{G}^{-1} G_{2}^{T} \\ G_{2} \bar{G}^{-1} G_{1}^{T} & G_{2} \bar{G}^{-1} G_{2}^{T}\end{array}\right]$.

The inverse $X^{+}$is now pseudoinverse of $X$. The cofactor matrix of this solution is $X^{+} X^{+^{T}}=\left(X^{T} X\right)^{+}$.

\subsubsection{Residuals}

The residuals in equation (12) are given by

$$
\left[\begin{array}{c}
v \\
v^{\prime}
\end{array}\right]=\left(I_{2 n}-X X^{g}\right)\left[\begin{array}{l}
l \\
l^{\prime}
\end{array}\right],
$$

where $X^{g}$ is any right weak generalised inverse (such as the inverses in equations (23), (26) and (29) above). The residuals do not depend on the particular choice of $X^{g}$ since $X X_{1}^{g}=X X_{2}^{g}$, where $X_{1}^{g}$ and $X_{2}^{g}$ are both right weak generalised inverses of $X$ (Boullion and Odell, 1971, p. 8).

The variance of the fit between the altimeter measurements and the $\mathrm{SSH} /$ orbit model is given by

$\sigma^{2}=\frac{\left(v^{T} v+v^{T} v^{\prime}\right)}{n-u+r}$.

It includes both $\mathrm{SSH}$ variations from the mean surface and measurement error.

\subsection{Multiple repeat cycles - Missing data}

We consider now the most general case of the crossover problem, where we have multiple repeat cycles but a certain percentage of data are missing in any cycle. The multiple repeat cycle solution with complete data is given in the Appendix.

It will be assumed that the satellite retraces the identical ground track in each repeat cycle, so that the crossovers refer to a fixed net of geographic locations. (Where there are significant across-track excursions from an exactly repeating ground track, it may be necessary to make geoid slope corrections of the kind discussed by Wang and Rapp (1991) and Rapp et al. (1994).)

The subscript notation introduced in section 2.2 will now be extended by the use of a further subscript to indicate the number of the repeat cycle. Let $l_{i}$ now be the $n$-vector of ascending altimeter crossover measurements in the $i$ th repeat cycle, and $l_{i}^{\prime}$ the corresponding vector of descending crossover measurements. The pair of measurements in this cycle at the $j$ th crossover point will be denoted by $l_{i j}$ and $l_{i j}^{\prime}$.

The measurements $l_{i j}, l_{i j}^{\prime}$ are related to the mean $\mathrm{SSH}$ $\bar{h}_{j}$ at the $j$ th location by

$l_{i j}=\bar{h}_{j}+A_{j} x_{i}+v_{i j}$

$l_{i j}^{\prime}=\bar{h}_{j}+A_{j}^{\prime} x_{i}+v_{i j}^{\prime}$,

where $x_{i}$ is the $u$-vector of orbit model parameters for repeat cycle $i, A_{j}, A_{j}^{\prime}$ are row vectors of model coefficients for the radial orbit error, for ascending and descending measurements, respectively, and $v_{i j}, v_{i j}^{\prime}$ are error terms. have

Putting all $2 n$ measurements in cycle $i$ together, we

$l_{i}=\bar{h}+A x_{i}+v_{i}$

$l_{i}^{\prime}=\bar{h}+A^{\prime} x_{i}+v_{i}^{\prime}$,

equivalent to equation (12). This is the measurement set that gets repeated $N$ times.

Let $e_{N}$ be the $N$-vector, each of whose elements is a one, and let

$l=\operatorname{vec}\left(l_{1}, l_{1}^{\prime}, l_{2}, l_{2}^{\prime}, \ldots, l_{N}, l_{N}^{\prime}\right)$

$v=\operatorname{vec}\left(v_{1}, v_{1}^{\prime}, v_{2}, v_{2}^{\prime}, \ldots, v_{N}, v_{N}^{\prime}\right)$

$x=\operatorname{vec}\left(x_{1}, x_{2}, \ldots, x_{N}\right)$.

The structure of the multiple repeat cycle solution given in the Appendix can easily be made to accommodate missing data (though the compact formulation using Kronecker products is no longer possible). We will consider only the case of orbit constraints $e_{N}^{T} \otimes G_{2}^{T} \cdot x=0$ (on average, the orbits are made to be free of GCE), since the solution with these constraints is the most feasible to calculate and other solutions can readily be obtained from this one.

Let $E_{i}$ be a diagonal $(n, n)$-matrix obtained from the $(n, n)$ identity matrix by placing a zero on the main diagonal wherever a crossover pair of measurements is 
missing in the $i$ th repeat cycle. Missing measurements in the observation vectors $l_{i}, l_{i}^{\prime}$ are filled out with zeroes, so that all vectors $l_{i}, l_{i}^{\prime}$ are of the same length $n$, and the $j$ th components of these vectors refer to the $j$ th crossover point, as before.

Let $A_{i}=E_{i} A$, replacing the rows of $A$ at missing data points for cycle $i$ with zeroes, and similarly $A_{i}^{\prime}=E_{i} A^{\prime}$. Putting all measurements for cycle $i$ together, we have (cf. equation (34)):

$\begin{aligned} l_{i} & =E_{i} \bar{h}+A_{i} x_{i}+v_{i} \\ l_{i}^{\prime} & =E_{i} \bar{h}+A_{i}^{\prime} x_{i}+v_{i}\end{aligned}$

(so that residuals corresponding to missing measurements also become zero). Let $n_{i}=$ trace $\left(E_{i}\right)$ be the number of pairs of data present in cycle $i$.

The contribution cycle $i$ makes to the normal equations is

$\left[\begin{array}{cc}2 E_{i} & A_{i}+A_{i}^{\prime} \\ \left(A_{i}+A_{i}^{\prime}\right)^{T} & A_{i}^{T} A_{i}+A_{i}^{\prime T} A_{i}^{\prime}\end{array}\right]\left[\begin{array}{c}\bar{h} \\ x_{i}\end{array}\right]=\left[\begin{array}{c}l_{i}+l_{i}^{\prime} \\ A_{i}^{T} l_{i}+A_{i}^{\prime T} l_{i}^{\prime}\end{array}\right]$.

The normal equation matrix for all $N$ cycles is

$$
\left[\begin{array}{cccc}
2 \sum_{i=1}^{N} E_{i} & A_{1}+A_{1}^{\prime} & \cdots & A_{N}+A_{N}^{\prime} \\
\left(A_{1}+A_{1}^{\prime}\right)^{T} & A_{1}^{T} A_{1}+A_{1}^{\prime T} A_{1}^{\prime} & \cdots & 0 \\
\left(A_{2}+A_{2}^{\prime}\right)^{T} & 0 & \cdots & 0 \\
\vdots & \vdots & \vdots & \vdots \\
\left(A_{N}+A_{N}^{\prime}\right)^{T} & 0 & \cdots & A_{N}^{T} A_{N}+A_{N}^{\prime T} A_{N}^{\prime}
\end{array}\right]
$$

corresponding to the full-data normal equation matrix in equation (43).

This matrix can be reduced by eliminating $\bar{h}$ to a matrix $N_{22}$ made up of $N$ by $N(u, u)$ sub-matrices. The diagonal blocks of $N_{22}$ are given by

$n_{i i}=A_{i}^{T} A_{i}+A_{i}^{\prime T} A_{i}^{\prime}-\frac{1}{2}\left(A_{i}+A_{i}^{\prime}\right)^{T} D\left(A_{i}+A_{i}^{\prime}\right)$

$(i=1,2, \ldots, N)$, and the off-diagonal blocks by

$n_{i j}=-\frac{1}{2}\left(A_{i}+A_{i}^{\prime}\right)^{T} D\left(A_{j}+A_{j}^{\prime}\right)$

$(i, j=1,2, \ldots, N, i \neq j)$, with

$D=\left(\sum_{i=1}^{N} E_{i}\right)^{-1}$.

The right-hand side of the normal equations may similarly be reduced to

$f=\left[\begin{array}{c}A_{1}^{T} l_{1}+A_{1}^{\prime T} l_{1}^{\prime}-\frac{1}{2}\left(A_{1}+A_{1}^{\prime}\right)^{T} D \sum_{i=1}^{N}\left(l_{i}+l_{i}^{\prime}\right) \\ A_{2}^{T} l_{2}+A_{2}^{\prime T} l_{2}^{\prime}-\frac{1}{2}\left(A_{2}+A_{2}^{\prime}\right)^{T} D \sum_{i=1}^{N}\left(l_{i}+l_{i}^{\prime}\right) \\ \vdots \\ A_{N}^{T} l_{N}+A_{N}^{\prime T} l_{N}^{\prime}-\frac{1}{2}\left(A_{N}+A_{N}^{\prime}\right)^{T} D \sum_{i=1}^{N}\left(l_{i}+l_{i}^{\prime}\right)\end{array}\right]$

The reduced normal equations with orbit constraints $e_{N}^{T} \otimes G_{2}^{T} \cdot x=0$ can be solved for the vector of orbit parameters: $\left[\begin{array}{l}x \\ k\end{array}\right]=\left[\begin{array}{cc}N_{22} & e_{N} \otimes G_{2} \\ e_{N}^{T} \otimes G_{2}^{T} & 0\end{array}\right]^{-1}\left[\begin{array}{l}f \\ 0\end{array}\right]$,

in which $k$ is an $r$-vector of Lagrange multipliers. The solution for the mean SSHs follows:

$\bar{h}=\frac{1}{D} \sum_{i=1}^{N}\left\{l_{i}+l_{i}^{\prime}-\left(A_{i}+A_{i}^{\prime}\right) x_{i}\right\}$

(cf. equation (48)).

The residuals are obtained in the usual way:

$v_{i}=l_{i}-E_{i} \bar{h}-A_{i} x_{i}$

$v_{i}^{\prime}=l_{i}^{\prime}-E_{i} \bar{h}-A_{i}^{\prime} x_{i}^{\prime}$.

These residuals also satisfy the conditions in equations (54) and (55).

The variance of the fit of the data to the crossover adjustment model is given by

$\sigma^{2}=\frac{\sum_{i=1}^{N}\left(v_{i}^{T} v_{i}+v_{i}^{\prime T} v_{i}^{\prime}\right)}{2 \sum_{i=1}^{N} n_{i}-n-N u+r}$.

\section{Data}

The crossover adjustment method, with orbit constraints, outlined immediately above, has been applied to the first 108 ten-day cycles of T/P data, with cycle 1 starting on 23 September 1992 and cycle 108 finishing on 30 August 1995. The data used were taken from the Merged Geophysical Data Records (MGDRs) distributed by the Physical Oceanography Distributed Active Archive Center (PO.DAAC) (Benada, 1993). These data contain measurements made with the NASA Radar Altimeter (ALT) and with the CNES experimental Solid State Al-timeter (SSALT). SSALT was on for the full duration of cycles 20,31, 41, 55, 65, 79, 91, 97 and 103 and for $10 \%$ of the time during cycles $1-16$, i.e. during the 'verification phase'; ALT was on for the remainder. We have used measurements from both altimeters, correcting SSALT ranges for the $15.4 \mathrm{~cm}$ relative bias reported by Christensen et al. (1994).

The measured altimeter ranges given by the MGDRs were corrected in the usual way, for electromagnetic bias (or significant wave height, using the ToPEX formula), for the wet and dry components of the tropospheric delay (using for the former, corrections obtained from the TOPEX Microwave Radiometer, TMR), and for the ionospheric delay (albeit without smoothing the TOPEX dual-frequency ionospheric correction). The NASA orbits were used to obtain sea surface heights from the altimeter ranges; these heights were then further corrected for tidal effects and inverse barometer effect (with ECMWF atmospheric pressure corrected for the S2 atmospheric tide, in the way suggested by Ray (1994)). For the tidal corrections we have used the Schwiderski model as given on the MDGRs, enhanced by the correction model given by Schrama and Ray (1994), but omitting their T/P-derived $S a$ and $S s a$ terms, 
since they include in these terms annual and semi-annual non-tidal effects we wish to see show up in our results. The four parameter model developed by Gaspar et al. (1994) has been used for correcting the sea-state bias (with different correction coefficients for the TOPEX and Poseidon altimeters). The new calibration value of sigma0 recommended by Callahan et al. (1994) has been used in calculating the wind speed contribution to the sea-state bias. The observed SSHs have not been adjusted for crosstrack geoid gradients.

Bad data were eliminated by referring to the appropriate flag settings (Benada, 1993). A certain amount of further editing was done to remove isolated points and short isolated sections of data, and to remove sections of data with implausible gradients or discontinuities. The measured data in each pass were then interpolated onto a fixed along-track grid (with points about $7 \mathrm{~km}$ apart), using a Gaussian filter with a halfmaximum width of about $50 \mathrm{~km}$. Crossover heights were then interpolated at fixed crossover points wherever the crossover point was straddled by pairs of gridded ascending and descending measurements (N. White, personal communication).

Data were only selected within the latitude band of $65^{\circ} \mathrm{N}$ to $65^{\circ} \mathrm{S}$, primarily to avoid problems with seasonal sea ice in the Southern hemisphere. In a further data editing step, crossover points were eliminated altogether if they contained less than $60 \%$ valid crossover measurement pairs; the 2165 points eliminated in this way tended to have considerably fewer than 64 pairs. A further small number of points were eliminated if the within-cycle root mean square crossover differences exceeded $0.40 \mathrm{~m}$ - removing a further 20 points, mostly in close proximity to the coast. What remained were a total of 5527 crossover points (out of a total 7712 crossover points represented in the data), with a total of 492727 pairs of measurements.

The orbit model used in the crossover adjustment is that of equation (1), with $l_{\max }=2$, i.e., with $\Delta r_{n g}$ contributing 5 coefficients and $\Delta r_{g} 21$ coefficients. Hence the observation equations (53) contain $8335[5527+108$ $(5+21)$ ] unknowns, with a rank defect that requires 9 constraint equations.

For the least squares solution for the SSHs $\bar{h}$ and orbit parameters $x$ the solution algorithm of sub-section 2.6 was implemented, using a computer program developed within the Matlab technical computing environment and executed on a Sun SPARC-20/61 computer workstation. The crossover solution of the full 108 cycles took approximately $400 \mathrm{CPU}$ seconds on the SPARC 20/61, using maximum array requirements of some $150 \mathrm{Mb}$ of memory, but most of the solution execution needed about $80 \mathrm{Mb}$.

\section{Results}

Our results include maps of mean sea surface height, of deviations of the sea surface from the mean, and of SSH variability, not very much different from those produced by other investigators (as reported, for example, in the special section on T/P in the December 1994 issue of Journal of Geophysical Research). There is one aspect of our solution, however, that does warrant attention. Our solution constrained the orbit errors, making the average orbit error over the 108 cycles we have processed equal to zero. The orbit errors for individual cycles need not be zero; the errors for individual cycles may be mapped spatially using equation (49), of expressed as surface spherical harmonic coefficients (of degree less than, or equal to, $\left.l_{\max }\right)$ by $\left(G_{2}^{T} G_{2}\right)^{-1} G_{2}^{T} x_{i}$. These coefficients (nine of them for $l_{\max }=2$ ) have been plotted against cycle number as a convenient means of understanding the solution.

In Figure 2 we show only the results for the low degree zonal terms. The cycle-by-cycle results are shown as solid lines with the shaded region representing the $3 \sigma$ level uncertainty derived from the scaled cofactor matrix of the crossover solution - see equation (19). The variations of the coefficients have a clear periodicity and we have computed a least squares fit to these data, using terms comprising of a bias, linear trend and annual, semi-annual and bi-annual periods. This best-fit curve is also shown as a solid line in Figure 2.

All low degree zone harmonics $\left(C_{100}, C_{10}\right.$ and $\left.C_{20}\right)$ contain a clear annual period, and a less obvious, though still significant, semi-annual period. The asymmetry between northern and southern hemispheres is evident, with the northern hemisphere having a stronger annual signal.

The question is 'Can we interpret these variations?' To address some of the issues, we compare the altimeter results with output from a numerical ocean circulation model. We have used the Hamburg Large Scale Geostrophic Ocean General Circulation Model (LSG) (Hasselmann, 1982; Maier-Reimer et al., 1993) for this comparison. The LSG model was initialised from a 10,000 year run and our results are taken from an average of 6 years of model output using a timestep of 10 days and with the model forced by wind fields of Hellerman and the Rosenstein [1983], freshwater fluxes and relaxation to the Levitus [1982] seasonal temperature field. The LSG model output was interpolated to the $\mathrm{T} / \mathrm{P}$ crossover grid locations and spherical harmonic coefficients computed in a similar fashion to the altimeter results. Further details of the LSG work and other model results are given in Bindoff et al., (1995).

The variation in $C_{00}$, i.e., in mean sea level, must be due, in part, to changes in the mass balance of the ocean caused by evaporation, precipitation and river run-off. However, the dominant amplitude of the annual signal of $C_{00}$ in Figure 2 is caused by the use of the inverse barometer correction (see also Minster et al., 1995; Nerem, 1995). The linear trend in $C_{00}$ gives an estimate of sea level rise, after correction for the drift in the altimeter range calibration - our estimates of 3-4 $\mathrm{mm} / \mathrm{yr}$ are similar to the findings of Nerem (1995), Minster et al. (1995) and others.

Figure 3 gives a comparison of the LSG model results (dashed line) and the T/P crossover solution. The amplitudes of the $C_{10}$ and $C_{20}$ terms are remarkably consistent, within $1 \mathrm{~cm}$ of each other, but the phases 
show differences of 10-25 days. The most important physics, acting on the time and space scales contributing to the sea level changes, is evident. Both $C_{10}$ and $C_{20}$ terms are primarily driven by the exchange of heat from the atmosphere to the oceanic mixed layer. The dominant annual cycle is caused by steric expansion of the mixed layer (the first $100 \mathrm{~m}$ or so) of the ocean, responding to the seasonal cycle in solar heating. This is seen in the $C_{10}$ term, for instance, by the time of the maximum sea level, about October in the northern hemisphere. The contribution of the winds to the barotropic response of the ocean is a contributing factor to the observed phase differences but these results are more fully described in Bindoff et al., (1995).

While the interpretation of our results in Figures 2 and 3 is still preliminary and far from complete, what the results do clearly show is that the GCE in our solution belongs to the longs-wavelength, long-period components of the ocean signal, rather than being a genuine part of the orbit error.

\section{Conclusion}

Presented in this paper is consistent, coherent strategy for carrying out a satellite altimetry crossover adjustment problem, in which the rank defect of the estimation problem and the existence of geographically correlated error (GCE) are explicitly recognised. Solutions are presented which place the GCE either in the orbits or in the mean sea surface heights. Our results for the first 108 cycles of Topex/PoseIDon show that the GCE, in the past often taken up as orbit error, is part of the ocean signal. Preliminary results with the same crossover adjustment method applied to Geosat and ERS-1 data indicate a GCE made up in part of orbit error, but also in part of the same long-wavelength and long-period variations of the ocean surface to be seen in Figure 2. In the case of T/P the orbits are so good there is little need to carry out a crossover adjustment, but it remains a useful thing to do for Geosat ad ERS-1 (and possibly also for the ERS-2 and Geosat Follow-On missions). In addition, our crossover solution strategy can readily be

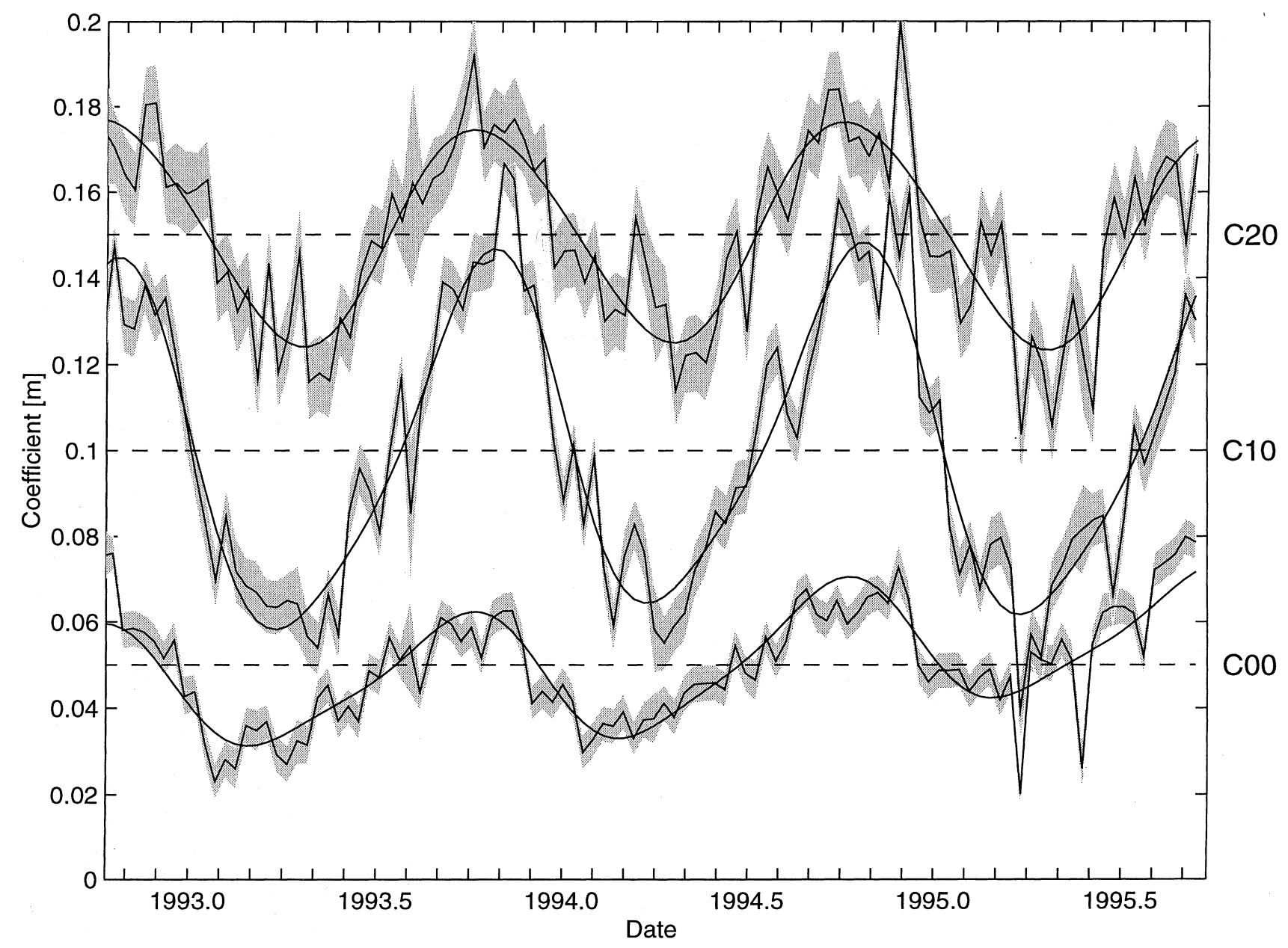

Fig. 2. Coefficients (in metres) for T/P cycles 1 to 108 of the three zonal (unnormalised) spherical harmonics that form the geographically correlated error associated with the radial orbit error model of equation (3), with $l_{\max }=2$. The results for each cycle are shown as a solid line with the shaded region representing their formal

uncertainties - a $3 \sigma$ level estimate derived from the scaled cofactor matrix of the crossover solution. A best-fitting curve, comprising a bias, linear trend, annual, semi-annual, semi-annual and bi-annual terms, is superimposed over the cycle-by-cycle results. 
extended to the crossover adjustment of data from more than one mission (for example, T/P and ERS-1). It can be extended, also, to include the estimation of residual ocean tide signals and the coefficients of empirical seastate bias models.

\section{A Multiple repeat cycles - complete data}

\section{A.1 Formulation}

For completeness, we give the case where there are data from $N$ complete repeat cycles of crossover data, i.e., wherever data are present at a particular crossover, a full set of $N$ ascending altimeter measurements and $N$ corresponding descending measurements are present.

Let $l_{i}$ be the $n$-vector of ascending altimeter crossover measurements in the $i$ th repeat cycle, and $l_{i}^{\prime}$ the corresponding vector of descending crossover measurements. The complete pair of measurements in this cycle at the $j$ th crossover point will be denoted by $l_{i j}$ and $l_{i j}^{\prime}$.

The measurements $l_{i j}, l_{i j}^{\prime}$ are related to the mean SSH $\bar{h}_{j}$ at the $j$ th location by equation (33) and this basic set of equations for cycle $i$ gets repeated $N$ times.
Let $e_{N}$ be the $N$-vector, each of whose elements is a one, and let

$l=\operatorname{vec}\left(l_{1}, l_{1}^{\prime}, l_{2}, l_{2}^{\prime}, \ldots, l_{N}, l_{N}^{\prime}\right)$

$v=\operatorname{vec}\left(v_{1}, v_{1}^{\prime}, v_{2}, v_{2}^{\prime}, \ldots, v_{N}, v_{N}^{\prime}\right)$

$x=\operatorname{vec}\left(x_{1}, x_{2}, \ldots, x_{N}\right)$.

The linear model relating all $2 \mathrm{Nn}$ measurements to the $n+N u$ unknown ( $n$ mean SSHs and $u$ orbit parameters for each repeat cycle) is

$$
\begin{aligned}
l= & {\left[e_{N} \otimes\left[\begin{array}{l}
I_{n} \\
I_{n}
\end{array}\right], I_{N} \otimes\left[\begin{array}{l}
A \\
A^{\prime}
\end{array}\right]\right]\left[\begin{array}{l}
\bar{h} \\
x
\end{array}\right]+v } \\
& =X\left[\begin{array}{l}
\bar{h} \\
x
\end{array}\right]+v
\end{aligned}
$$

where ' $\otimes$ ' is the Kronecker or tensor product (Rao and Mitra, 1971; Brewer, 1978).

The rank defect in this linear model has not been changed by repeating the measurement sequence. The nullspace is spanned by

$$
G=\left[\begin{array}{c}
G_{1} \\
e_{N} \otimes \\
G_{2}
\end{array}\right]
$$

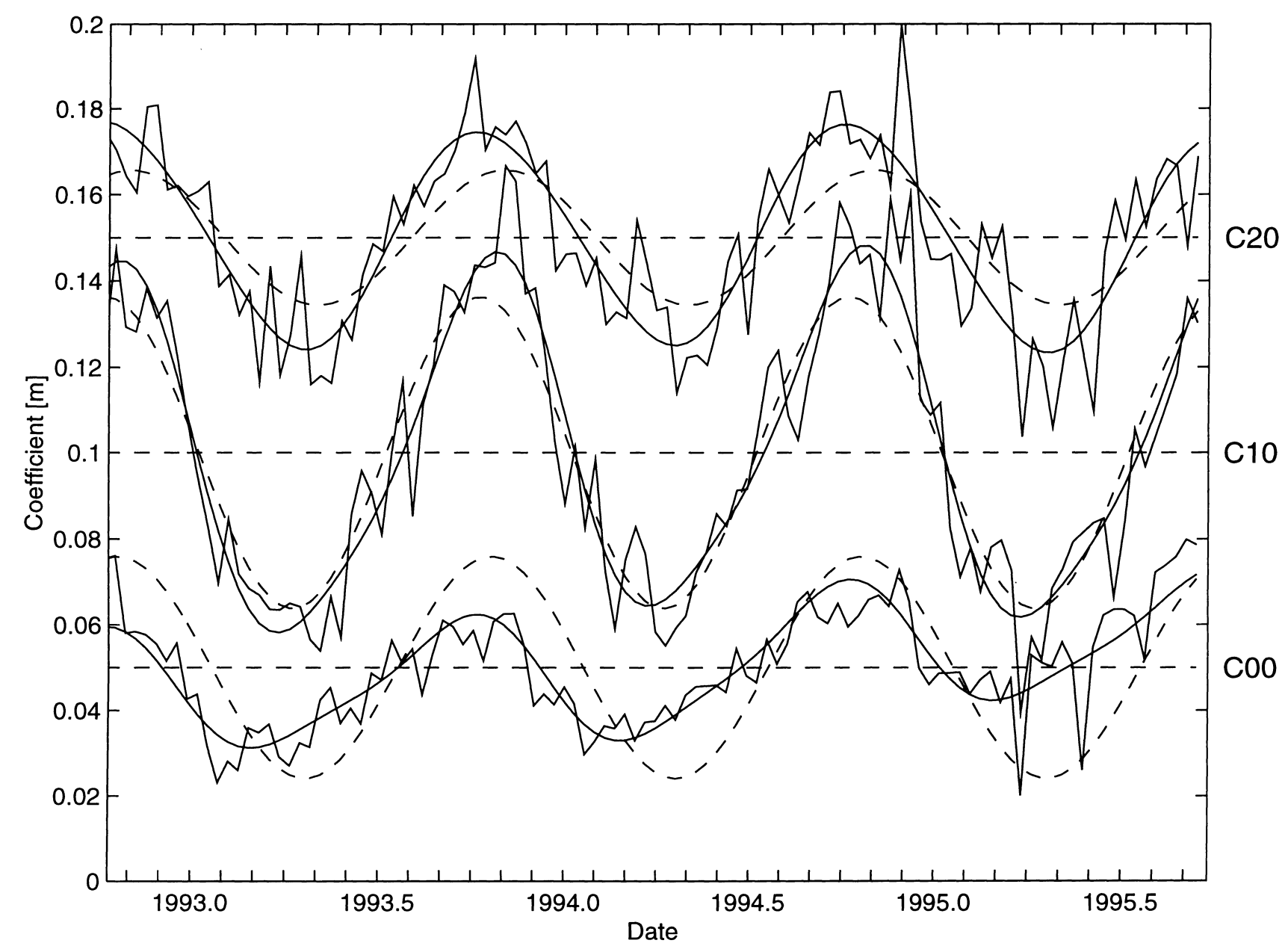

Fig. 3. The same coefficients (in metres) for T/P cycles 1 to 108 of the three zonal (unnormalised) spherical harmonics and the best-fitting curve as in Figure 2, with the numerical ocean model results indicated by the dashed lines. 
and

$$
\begin{aligned}
X G & =\left[e_{N} \otimes\left[\begin{array}{l}
I_{n} \\
I_{n}
\end{array}\right], I_{N} \otimes\left[\begin{array}{l}
A \\
A^{\prime}
\end{array}\right]\right]\left[\begin{array}{c}
G_{1} \\
e_{N} \otimes G_{2}
\end{array}\right] \\
& =e_{N} \otimes\left[\begin{array}{l}
G_{1}+A G_{2} \\
G_{1}+A^{\prime} G_{2}
\end{array}\right] \\
& =0_{(2 N n, r)},
\end{aligned}
$$

and $\operatorname{rank}(G)=\operatorname{rank}\left(\left[\begin{array}{c}G_{1} \\ e_{N} \otimes G_{2}\end{array}\right]\right)=r$, as before.

The normal equations corresponding to equation (40) are

$X^{T} X\left[\begin{array}{l}\bar{h} \\ x\end{array}\right]=X^{T} l$

where

$X^{T} X=\left[\begin{array}{cc}2 N I_{n} & e_{N}^{T} \otimes\left(A+A^{\prime}\right) \\ e_{N} \otimes\left(A+A^{\prime}\right)^{T} & I_{N} \otimes\left(A^{T} A+A^{\prime T} A^{\prime}\right)\end{array}\right]$.

\section{A.2 Orbital constraints}

We follow much the same procedure as in section 2.5.1 above. The normal equation matrix $X^{T} X$ in equation (42) can be reduced to

$$
\left[\begin{array}{cc}
2 N I_{n} & e_{N}^{T} \otimes\left(A+A^{\prime}\right) \\
0 & N_{22}
\end{array}\right]
$$

where

$$
\begin{aligned}
N_{22}= & \left(I_{N}-\frac{1}{N} e_{N} e_{N}^{T}\right) \otimes\left(A^{T} A+A^{\prime T} A^{\prime}\right) \\
& +\frac{1}{N} e_{N} e_{N}^{T} \otimes \frac{1}{2}\left(A-A^{\prime}\right)^{T}\left(A-A^{\prime}\right) .
\end{aligned}
$$

The product of the two terms summed in this equation is zero, and $\left(I_{N}-\frac{1}{N} e_{N} e_{N}^{T}\right)$, being a projection matrix, is its own pseudoinverse. In consequence, the pseudoinverse of $N_{22}$ is the sum of the pseudoinverses of the two terms (Boullion and Odell, 1971):

$$
\begin{aligned}
N_{22}^{+}=\left(I_{N}\right. & \left.-\frac{1}{N} e_{N} e_{N}^{T}\right) \otimes\left(A^{T} A+A^{\prime T} A^{\prime}\right)^{-1} \\
& +\frac{1}{N} e_{N} e_{N}^{T} \otimes Q_{x},
\end{aligned}
$$

where $Q_{x}$ is given by equation (19).

Hence

$$
\begin{aligned}
& x=N_{22}^{+}\left\{\left(I_{N}-\frac{1}{N} e_{N} e_{N}^{T} \otimes\left[\begin{array}{c}
A \\
A^{\prime}
\end{array}\right]^{T}\right.\right. \\
&\left.\left.+\frac{1}{N} e_{N} e_{N}^{T}\right) \otimes \frac{1}{2}\left[\begin{array}{c}
A-A^{\prime} \\
-\left(A-A^{\prime}\right)
\end{array}\right]^{T}\right\} l \\
&=\left(I_{N}-\frac{1}{N} e_{N} e_{N}^{T}\right) \otimes\left(A^{T} A+A^{\prime T} A^{\prime}\right)^{-1}\left[\begin{array}{l}
A \\
A^{\prime}
\end{array}\right]^{T} l \\
&+\frac{1}{N} e_{N} e_{N}^{T} \otimes \frac{1}{2} Q_{x}\left[\begin{array}{c}
A-A^{\prime} \\
-\left(A-A^{\prime}\right)
\end{array}\right]^{T} l
\end{aligned}
$$

or, breaking this up into solutions for individual cycles,

$x_{i}=y_{i}-\frac{1}{N} \sum_{j=1}^{N} y_{j}+\frac{1}{N} \sum_{j=1}^{N} z_{j}$

where

$$
\begin{aligned}
y_{j} & =\left(A^{T} A+A^{\prime T} A^{\prime}\right)^{-1}\left(A^{T} l_{j}+A^{\prime T} l_{j}^{\prime}\right) \\
z_{j} & =\frac{1}{2} Q_{x}\left(A-A^{\prime}\right)^{T}\left(l_{j}-l_{j}^{\prime}\right)
\end{aligned}
$$

(cf. equation (20) for a single repeat cycle).

The mean sea surface heights now follow by substituting $x$ into the first row of the normal equations (42):

$\bar{h}=\frac{1}{2 N}\left\{e_{N}^{T} \otimes\left[\begin{array}{l}I_{n} \\ I_{n}\end{array}\right]^{T} l-e_{N}^{T} \otimes\left(A+A^{\prime}\right) x\right\}$,

but this result can be simplified by noting that

$$
\begin{aligned}
e_{N}^{T} \otimes\left(A+A^{\prime}\right) x & =e_{N}^{T} \otimes \frac{1}{2}\left(A+A^{\prime}\right) Q_{x}\left[\begin{array}{c}
A-A^{\prime} \\
-\left(A-A^{\prime}\right)
\end{array}\right]^{T} l \\
& =e_{N}^{T} \otimes\left(A+A^{\prime}\right) z
\end{aligned}
$$

where $z=\operatorname{vec}\left(z_{1}, z_{2}, \ldots, z_{N}\right)$. The solution for the mean SSHs thus becomes

$\bar{h}=\frac{1}{N} \sum_{j=1}^{N} \frac{1}{2}\left\{l_{j}+l_{j}^{\prime}-\left(A+A^{\prime}\right) z\right\}$.

Finally, it is easily verified that $e_{N}^{T} \otimes G_{2}^{T} \cdot x=0$, as we require.

We thus see in equation (47) that the orbit errors $x_{i}$ for cycle $i$ depend on data for all cycles, but that the mean SSHs can be calculated as a simple mean of altimeter heights, corrected for orbit errors using parameters $z_{i}$ calculated from a single cycle.

The constraint $e_{N}^{T} \otimes G_{2}^{T} \cdot x=0$ says that, on average, the orbit are free of GCE; nevertheless, for any individual cycle it need not follow that $G_{2}^{T} x_{i}=0$. The amount by which the GCE within a cycle differs from the mean can be given its spatial expression by

$\Delta h_{i}=G_{1}\left(G_{2}^{T} G_{2}\right)^{-1} G_{2}^{T} x_{i}$.

\section{A.3 SSH constraints}

As before, we suggest that the easiest way to obtain mean SSHs and orbit parameters in which the GCE has been placed in the orbits, and in which none of it appears in $\bar{h}$, is through a transformation, as in equations (25) and (26).

For multiple repeat cycles the transformation from the orbit-constrained solution (or any other minimum constraint solution) to the solution putting the constraint into mean SSHs is

$$
T=I_{n+N u}-\left[\begin{array}{cc}
G_{1}\left(G_{1}^{T} G_{1}\right)^{-1} G_{1}^{T} & 0 \\
e_{N} \otimes G_{2}\left(G_{1}^{T} G_{1}\right)^{-1} G_{1}^{T} & 0
\end{array}\right] .
$$


In particular, the transformation for mean SSHs is $\bar{h}_{\text {SSH constrained }}=\left(I_{n}-G_{1}\left(G_{1}^{T} G_{1}\right)^{-1} G_{1}^{T}\right) \bar{h}_{\text {orbit constrained }}$,

a calculation which does not grow with the number of repeat cycles. Moreover, it is easy to see from equation (48) that conditions $G_{1}^{T} \bar{h}_{\mathrm{SSH}}$ constrained $=0$ is satisfied.

\section{A.4 Minimum norm solution}

In a similar way the constrained orbit solution may also be transformed to a minimum norm solution, by the transformation

$X^{+}=T X^{g}$,

where $X^{g}$ is any minimum constraint solution (right weak inverse), and where

$T=I_{n+N u}-G\left(G^{T} G\right)^{-1} G^{T}$

with $G$, the matrix spanning the nullspace of $X$ as given in equation (38).

Again, it is easily verified that $G^{T} X^{+}=G^{T} T X^{g}=0$.

\section{A.5 Residuals}

The residuals, for multiple repeat cycles, are given by something akin to equation (31)

$v=\left(I_{n+N u}-X X^{g}\right) l$.

The residuals for cycle $i$ can be calculated from

$v_{i}=l_{i}-\bar{h}-A x_{i}$

$v_{i}^{\prime}=l_{i}^{\prime}-\bar{h}-A^{\prime} x_{i}$

It bears repetition that the residuals do not depend on the choice of right weak inverse $X^{g}$.

The residuals, as always, are orthogonal to the least squares solution space, i.e. orthogonal to the column space of $X$, i.e. $X^{T} v=0$. Now,

$X^{T}=\left[\begin{array}{c}e_{N}^{T} \otimes\left[I_{n}, I_{n}\right] \\ I_{N} \otimes\left[A^{T}, A^{\prime T}\right]\end{array}\right]$,

so the sum of the residuals at any crossover point is zero:

$\sum_{i=1}^{N}\left(v_{i j}+v_{i j}^{\prime}\right)=0$.

Further, within each cycle

$A^{T} v_{i}+A^{\prime T} v_{i}^{\prime}=0$.

In particular this means (since a bias is a part of the orbit model)

$\sum_{j=1}^{n}\left(v_{i j}+v_{i j}^{\prime}\right)=0$ i.e., all residuals within a cycle also sum to zero. More generally, the residuals contain nothing (either spatially or temporally) that can be construed to be GCE.

Acknowledgement. We are grateful to Neil White, CSIRO Division of Oceanography, Hobart, Tasmania, for help with data preparation. Nathan Bindoff and Jörg-Olaf Wolff provided the results from the ocean modelling comparisons. This work is supported by the Australian Research Council (RC) and the University of Natal Research Fund (HvG).

\section{References}

Benada, R., PO.DAAC Merged GDR (Topex/Poseidon) Users Handbook, JPL D-11007, Jet Propulsion Laboratory, Pasadena, Calif., 1993.

Bertiger, W.I., Y.E. Bar-Sever, E.J. Christensen, E.S. Davis, J.R. Guinn, B.J. Haines, R.W. Ibanez-Meier, J.R. Jee, S.M. Lichten, W.G. Melbourne, R.J. Muellerschoen, T.N. Munson, Y. Vigue, S.C. Wu, T.P. Yunck, B.E. Schutz, P.A.M. Abusali, H.J. Rim, M.M. Watkins and P. Willis, GPS precise tracking of TOPEX/ POSEIDON: Results and implications, J. Geophys. Res., 99, 24449-24464, 1994.

Bindoff, N.L., R. Coleman, H. van Gysen and N.J. White, Comparison of seasonal sea-level signals from altimetry and ocean models, paper presented at International Association of the Physical Sciences of the Ocean (IAPSO) XXI General Assembly, Honolulu, Hawali, USA, August 5-12, 1995.

Blanc, F., P.Y. LeTraon and S. Houry, Reducing Orbit Error for a Better Estimate of Oceanic Variability from Satellite Altimetry, J. Atmos. Oceanic Technol., 12, 150-160, 1995.

Brewer, J.W., Kronecker products and matrix calculus in system theory, IEEE Trans. Circ. Syst., CAS-25(9), 772-781, 1978.

Boullion, T.L., and P.L. Odell, Generalized Inverse Matrices, Wiley, New York, 1971.

Callahan, P.S., D.W. Hancock and G.S. Hayne, New sigma0 calibration for the TOPEX altimeter, TOPEX/Poseidon Research News, 3, 28-30, 1994.

Chelton, D.B., and M.G. Schlax, Spectral characteristics of timedependent orbit errors in altimeter height measurements, J. Geophys. Res., 98, 12579-12600, 1993.

Christensen, E.J., B.J. Haines, S.J. Keihm, C.S. Morris, R.S. Norman, G.H. Purcell, B.G. Williams, B.C. Wilson, G.H. Born, M.E. Parke, S.K. Gill, C.K. Shum, B.D. Tapley, R. Kolenkiewicz and S. Nerem, Calibration of Topex/PoseIDon at Platform Harvest, J. Geophys. Res., 99, 24465-24485, 1994.

Colombo, O.L., Altimetry, Orbits and Tides, NASA Tech. Memo. 86180, NASA, Greenbelt MD, 1984.

Douglas, B.C., R.W. Agreen and D.T. Sandwell, Observing global ocean circulation with Seasat altimeter data, Mar. Geod., 8, 6789, 1984.

Engelis, T., On the simultaneous improvement of a satellite orbit and determination of sea-surface topography using satellite altimetry data, Manuscripta Geodaetica, 13, 180-190, 1988.

Fu, L.-L., and J. Vazquez, On correcting radial orbit errors for altimetric satellites using crossover analysis, J. Atmos. Oceanic Technol., 5, 466-471, 1988.

Gaspar, P., F. Ogor, P.-Y. Le Traon and O.-Z. Zanife, Estimating the sea state bias of the Topex and PoseIDON altimeters from crossover differences, J. Geophys. Res., 99, 24981-24994, 1994.

Haines, B.J., G.H. Born, R.G. Williamson and C.J. Koblinsky, Application of the GEM-T2 gravity model to altimetric satelite orbit computation, J. Geophys. Res., 99, 16237-16254, 1994.

Hasselmann, K., An ocean model for climate variability studies, Prog. Oceanography, 11, 69-92, 1982.

Heiskanen, W.A., and H. Moritz, Physical Geodesy, Freeman, San Fransisco, 1967. 
Hellerman, S., and M. Rosenstein, Normal monthly wind stress over the world ocean with error estimates, J. Phys. Oceanogr, 13, 1093-1104, 1983.

Houry, S., J.F. Minster, C. Broussier, K. Dominh, M.C. Gennero, A. Cazenave and P. Vincent, Radial orbit error reduction and mean sea surface computation from the Geosat altimeter data, J. Geophys. Res., 99, 4519-4531, 1994.

Kaula, W.M., Theory of Satellite Geodesy, Blaisdell, Waltham Mass., 1966

Leick, A., GPS Satellite Surveying, Wiley, New York, 1990.

Le Traon, P.Y., J. Stum, J. Dorandev, P. Gaspar and P. Vincent, Global statistical analysis of TOPEX and Poseidon data, J. Geophys. Res., 99, 24619-24631, 1994.

Levitus, S., Climatological Atlas of the World Ocean, NOAA Professional Paper 13, U.S. Department of Commerce, 1982.

Maier-Reimer, E., U. Mikolajewicz and K. Hasselmann, Mean circulation of the HAMBURG LSG OGCM and its sensitivity to the thermohaline surface forcing, J. Phys. Oceanogr., 23, 731-757, 1993.

Menard, Y., E. Jeansou and P. Vincent, Calibration of Topex/ PoseIdon altimeter at Lampedusa, J. Geophys. Res., 99, $24487-$ 24504, 1994.

Mesias, J.M., and P.T. Strub, An Inversion Method to Determine Ocean Surface Currents Using Irregularly Sampled Satellite Altimetry Data, J. Atmos. Oceanic Technol., 12, 830-849, 1995.

Miller, L., R. Cheney and J. Lillibridge, Blending ERS-1 altimetry and tide gauge data, Eos Trans. AGU, 74, 185, 197, 1993.

Minster, J.-F., C. Brossier and P. Rogel, Variation of the mean sea level from TOPEX/POSEIDON data, J. Geophys. Res., 100, (C12), 25,153-25,161, 1995.

Moore, P., and S. Ehlers, Orbital improvement of ERS-1 using dual crossover arc techniques with Topex/Poseidon, Manuscripta Geodaetica, 18, 249-262, 1993.

Moore, P., and D.A. Rothwell, A study of gravitational and nongravitational modelling errors in cross-over differences, Manuscripta Goedaetica, 15, 187-206, 1990.

Nerem, R.S., Measuring global mean sea level variations using TOPEX/POSEIDON altimeter data, J. Geophys. Res., 100, C12, 25,135-25,151, 1995.

Rao, C.R., and S.K. Mitra, Generalised Inverse of Matrices and its Applications, Wiley New York, 1971.

Rapp, R.H., Geos-3 data processing for the recovery of geoid undulations and gravity anomalies, J. Geophys. Res., 84, 3784 3792, 1979.

Rapp, R.H., Y. Yi and Y.M. Wang, Mean sea surface and geoid gradient comparisons with TOPEX altimeter data, J. Geophys. Res., 99, 24657-24667, 1994.

Ray, R.D., Atmospheric tides and the Topex/Poseidon meteorological corrections, TOPEX/Poseidon Research News, 3, 12 14, 1994.

Rosborough, G.W., Satellite orbit perturbations due to the geopotential, CSR-86-1, Center for Sapce Research, The University of Texas at Austin, 1986.

Schrama, E.J.O., The role of orbit errors in processing of satellite altimeter data, Netherlands Geod. Commn., Delft, 1989.

Schrama, E.J.O., Some remarks on several definitions of geographically correlated orbit errors: consequences for satellite altimetry, Manuscripta Geodaetica, 17, 282-294, 1992.
Schrama, E.J.O., and R.D. Ray, A preliminary tidal analysis of TopeX/Poseidon altimetry, J. Geophys. Res., 99, 24799-24808, 1994.

Schum, C.K., D.N. Yuan, J.C. Ries, J.C. Smith, B.E. Schutz, and B.D. Tapley, Precision orbit determination for the Geosat Exact Repeat Mission, J. Geophys. Res., 95, 2887-2898, 1990.

Stammer, D., and C. Wunsch, Preliminary assessment of the accuracy and precision of TOPEX/Poseidon altimeter data with respect to the large scale ocean circulation, J. Geophys. Res., 99, 24584-245604, 1994.

Tai, C.-K., On Determining the Large-Scale Ocean Circulation From Satellite Altimetry, J. Geophys. Res., 88, 9553-9565, 1983.

Tai, C.-K., Geosat crossover analysis in the Tropical Pacific 1. Constrained sinusoidal crossover adjustment, J. Geophys. Res., 93, 10621-10629, 1988.

Tai, C.-K., How to Observe the Gyre to Global-Scale Variability in Satellite Altimetry: Signal Attenuation by Orbit Error Removal, J. Atmos. Oceanic Technol., 8, 271-288, 1991.

Tai, C.-K., and L.-L. Fu, On crossover adjustment in satellite altimetry and its oceanographic implications, J. Geophys. Res., 91, 2549-2554, 1986.

Tai, C.-K., and C.A. Wagner, Along-track spectra of ocean seasonal cycle and their attenuation by various orbit error removal schemes in satellite altimetry, U.S. Dept. of Commerce, NOAA Memo. OES-010, Washington D.C., 16pp, 1994.

Tapley, B.D., J.C. Ries, G.W. Davis, R.J. Eanes, B.E. Schutz, C.K. Shum, M.M. Watkins, J.A. Marshall, R.S. Nerem, B.H. Putney, S.M. Klosko, S.B. Luthcke, D. Pavlis, R.G. Williamson and N.P. Zelensky, Precision orbit determination for ToPEX/ Poseidon, J. Geophys. Res., 99, 24383-24404, 1994.

ToPEX/PoseIdon Project, GDR User's Handbook, JPL D-8944, Jet Propulsion Laboratory, Calif., 1992.

Van Gysen, H., R. Coleman and B. Hirsch, Local Crossover Analysis of Exactly Repeating Satellite Altimeter Data, submitted to Manuscripta Geodaetica, 1995.

Wagner, C.A., Radial variations of a satellite due to gravitational errors: implications for satellite altimetry, J. Geophys. Res., 90, 3027-3036, 1985.

Wagner, C.A., and R.E. Cheney, Global sea level change from satellite altimetry, J. Geophys. Res., 97, 15607-15615, 1992.

Wagner, C.A., and C.K. Tai, Degradation of ocean signals in satellite altimetry due to orbit error removal processes, J. Geophys. Res., 99, 16255-16267, 1994.

Wang, Y.M., and R.H. Rapp, Geoid gradients for Geosat and Topex/PoseIDon repeat ground tracks, Department of Geodetic Science and Surveying, Ohio State University, Report No 408, 1991.

White, N.J., R. Coleman, J.A. Church, P.J. Morgan and S.J. Walker, A southern hemisphere verification for the TOPEX/ Poseidon altimeter mission, J. Geophys. Res., 99, 24505-24516, 1994.

Wunsch, C., and V. Zlotnicki, The accuracy of altimetric surfaces, Geophys.J.Roy.Astron.Soc., 78, 795-808, 1984.

Wunsch, C., and E.M. Gaposchkin, On using satellite altimetry to determine the general circulation of the oceans with application to geoid improvement, Rev. Geophys. Space Phys., 18, 725-745, 1980 . 